\title{
In utero exposure to transient ischemia- hypoxemia promotes long-term neurodevelopmental abnormalities in male rat offspring
}

\author{
Arvind Palanisamy, ${ }^{1,2}$ Tusar Giri, ${ }^{1}$ Jia Jiang, ${ }^{1}$ Annie Bice, ${ }^{3}$ James D. Quirk, ${ }^{3}$ Sara B. Conyers, ${ }^{4}$ \\ Susan E. Maloney, ${ }^{4}$ Nandini Raghuraman, ${ }^{2}$ Adam Q. Bauer, ${ }^{3}$ Joel R. Garbow, ${ }^{3}$ and \\ David F. Wozniak 4,5 \\ ${ }^{1}$ Department of Anesthesiology, ${ }^{2}$ Department of Obstetrics and Gynecology, ${ }^{3}$ Mallinckrodt Institute of Radiology, and \\ ${ }^{4}$ Department of Psychiatry, and ${ }^{5}$ Taylor Family Institute for Innovative Psychiatric Research, Washington University School \\ of Medicine, St. Louis, Missouri, USA.
}

\begin{abstract}
The impact of transient ischemic-hypoxemic insults on the developing fetal brain is poorly understood despite evidence suggesting an association with neurodevelopmental disorders such as schizophrenia and autism. To address this, we designed an aberrant uterine hypercontractility paradigm with oxytocin to better assess the consequences of acute, but transient, placental ischemia-hypoxemia in term pregnant rats. Using MRI, we confirmed that oxytocin-induced aberrant uterine hypercontractility substantially compromised uteroplacental perfusion. This was supported by the observation of oxidative stress and increased lactate concentration in the fetal brain. Genes related to oxidative stress pathways were significantly upregulated in male, but not female, offspring 1 hour after oxytocin-induced placental ischemia-hypoxemia. Persistent upregulation of select mitochondrial electron transport chain complex proteins in the anterior cingulate cortex of adolescent male offspring suggested that this sex-specific effect was enduring. Functionally, offspring exposed to oxytocin-induced uterine hypercontractility showed malespecific abnormalities in social behavior with associated region-specific changes in gene expression and functional cortical connectivity. Our findings, therefore, indicate that even transient but severe placental ischemia-hypoxemia could be detrimental to the developing brain and point to a possible mitochondrial link between intrauterine asphyxia and neurodevelopmental disorders.
\end{abstract}

Conflict of interest: The authors have declared that no conflict of interest exists.

Copyright: (ㄷ) 2020, American Society for Clinical Investigation.

Submitted: September 3, 2019

Accepted: April 22, 2020

Published: May 21, 2020

Reference information: /CI Insight. 2020;5(10):e133172.

https://doi.org/10.1172/jci.

insight.133172.

\section{Introduction}

Epidemiological studies suggest a link between obstetric complications and neurodevelopmental disorders (1-5), but the underlying biological mechanisms remain largely unexplored. The consequences of severe birth asphyxia are well understood both in human and in animal studies (6-12), but the neurodevelopmental impact of transient and less severe hypoxemic insults, which are more common during labor and delivery, is poorly understood. For example, aberrant uterine contractility (tachysystole and tetanic uterine contraction) can often lead to transient fetal distress, which may or may not necessitate emergent delivery. These episodes are common, occurring in approximately $5 \%-10 \%$ of all laboring women $(13,14)$, especially when oxytocin (OXT) is used for induction and augmentation of labor. It is widely presumed that these episodes are usually benign because the surrogate markers of fetal well-being, such as umbilical arterial lactate and $\mathrm{pH}$, are typically unaffected. Though this is reassuring, it is not known whether these transient ischemic-hypoxemic episodes have an impact on the fetal brain, arguably the most important organ of interest. This knowledge is critical because of the exquisite sensitivity of the fetal brain to hypoxia and its susceptibility to oxidative stress because of the lack of robust antioxidant defense mechanisms (15). Current animal models that examine this question are contrived because the procedures devised to create placental ischemia-hypoxemia are very invasive (e.g., uterine artery ligation or occlusion) $(16,17)$, require prolonged anesthesia, and rarely reflect the transient and reversible ischemic-hypoxemic insults that are far more common during labor and delivery. Here, we created a pragmatic and translationally relevant pregnant rat model by stimulating uterine 
hypercontractility with OXT. We show that such uterine hypercontractility causes transient placental ischemia-hypoxemia, significant oxidative stress in the fetal brain, enduring brain region-specific dysfunction of the mitochondrial electron transport chain complex, abnormal cortical functional connectivity, and subtle neurobehavioral abnormalities, especially in the adolescent male offspring.

\section{Results}

OXT-induced uterine hypercontractility causes substantial placental ischemia. Though aberrant uterine contractility is known to cause uteroplacental hypoperfusion in pregnant women, it was unclear whether this could be replicated in a pregnant rat model. Therefore, we addressed this by quantifying the effect of OXT-induced uterine hypercontractility on placental perfusion in term pregnant Sprague-Dawley rats (E21) with dynamic contrast-enhanced MRI (Figure 1, A and B). Placental uptake of Dotarem contrast was approximately 45\% lower after OXT-induced hypercontractility, indicating a significant decrease in uteroplacental blood flow. Next, we assessed whether the impaired uteroplacental perfusion was accompanied by placental hypoxemia with effective transverse relaxation rate constant $\left(\mathrm{R} 2^{*}\right)$ mapping in a separate set of experiments (Figure $\left.1 \mathrm{C}\right)$. Thirty minutes after OXT, there was a significant change in the junctional zone contrast such that the placentas appeared completely bright with a significantly lower R2*. Though hypoxic placenta is expected to demonstrate a higher $\mathrm{R} 2 *$, this change in junctional zone contrast is the effect of a longer T2 (and/or T2*, the "natural" transverse relaxation time constant; $\mathrm{T} 2 *=1 / \mathrm{R} 2^{*}$ ), which would reasonably be explained by a significant decrease in iron (or deoxyhemoglobin). This suggested that these placentas were either not receiving much blood or having it expelled out of them by the tetanic uterine contraction. Collectively, our placental imaging data show that OXT-induced hypercontractility causes substantial impairment of placental perfusion.

OXT-induced uterine hypercontractility is associated with oxidative stress in the fetal brain. Acute uteroplacental hypoperfusion could lead to fetal hypoxemia and affect the fetal brain. Consequently, we determined the impact of OXT-induced transient placental ischemia-hypoxemia on the developing brain by assaying for lactate and oxidative stress markers (Figure 2, A-D). Fetal brain lactate was significantly higher 4 hours after OXT versus saline (Figure 2A), suggesting that the impaired uteroplacental perfusion after OXT was accompanied by fetal hypoxemia. Because placental ischemia-hypoxemia induced by OXT is transient, we speculated that recovery from such an episode would be accompanied by oxidative stress. Therefore, we assayed the fetal cortex for biomarkers of oxidative stress 4 hours after OXT and observed a significant increase in 4-hydroxynonenal (a lipid peroxidation product; Figure 2B) and protein carbonyl (a protein oxidation product; Figure 2C) content in pups exposed to OXT-induced hypercontractility. Concomitantly, we detected a significant increase in the oxidized glutathione ratio in the fetal cortex after OXT, suggesting a decrease in antioxidant defense (Figure 2D). These findings supported our prediction that acute placental ischemia from OXT-induced aberrant uterine contractility will cause oxidative stress in the fetal brain.

Effect of OXT-induced uterine hypercontractility on the fetal cortical transcriptome. Because acute hypoxia and oxidative stress could induce transcriptomic changes in the developing brain, we assessed for this possibility by screening the fetal cortex 24 hours after OXT-induced uterine hypercontractility with unbiased RNA-Seq (Figure 3, A-D). Overall, 936 of the 12,660 transcripts assessed were differentially expressed (unadjusted $P<0.05$ ) after OXT-induced hypercontractility (heatmap in Supplemental Figure 1; supplemental material available online with this article; https://doi.org/10.1172/jci.insight.133172DS1). Notably, of the top 50 differentially expressed genes, 9 of them were mitochondrially encoded ( $m t-N d 2$, $m t-N d 1, m t-N d 3, m t-N d 4, m t-N d 4, m t-N d 5, m t-N d 6, m t-C y b, m t-A t p 6)$. With an FDR-adjusted $P$ less than or equal to 0.05 , only $m t-N d 2$ (mitochondrially encoded NADH dehydrogenase 2 in complex I) was significantly differentially expressed among the 936 transcripts. GO analyses revealed activation of multiple biological signaling pathways and molecular transporter activity, along with downregulation of DNA repair and nucleic acid binding activity (Figure 3, A and B). KEGG pathway analyses (Figure 3, C and D) revealed activation of calcium signaling mechanisms and downregulation of pathways, including the Fanconi anemia pathway, important for DNA repair and known to be downregulated after hypoxic stress $(18,19)$. Considering the increase in oxidative stress biomarkers, the increased expression of $m t-N d 2$ (essential for mitochondrial production of reactive oxygen species), and activation of calcium signaling mechanisms and downregulation of DNA repair pathways typically observed after hypoxic injury, we concluded that one of the major effects of OXT-induced hypercontractility on the fetal brain is severe hypoxic stress. Of note, there was no significant differential expression of genes in the OXT/OXT receptor (OXT/OXTR) signaling pathway (Supplemental Figure 2). 
A

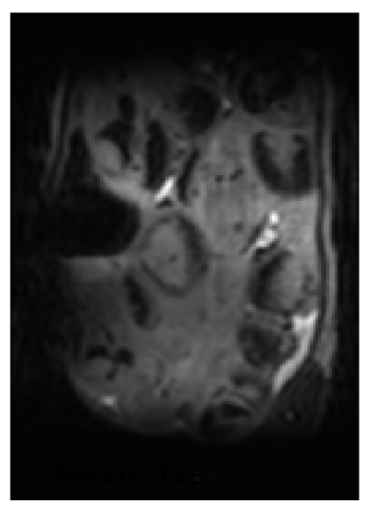

B

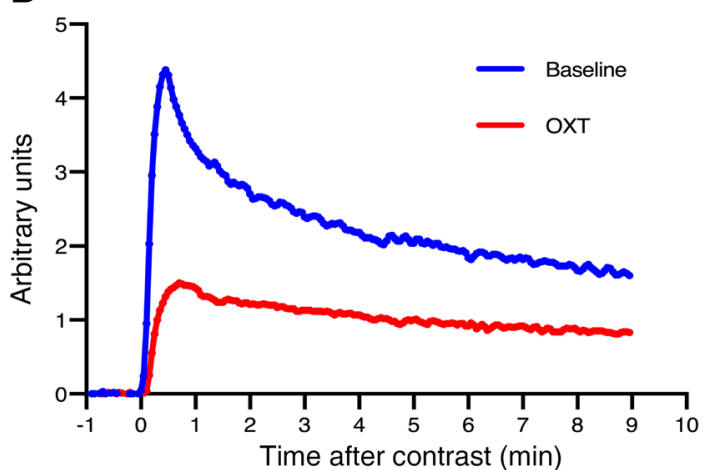

C After OXT FSE

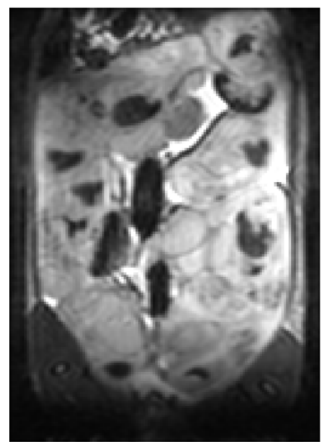

Time after contrast (min)

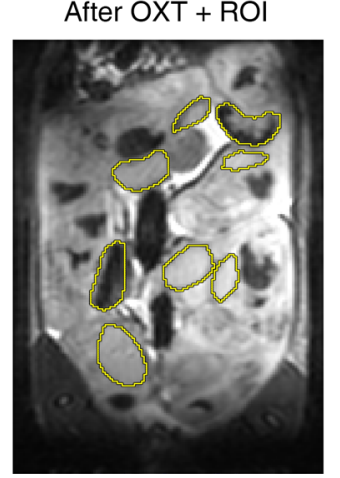

Baseline + IAUC overlay
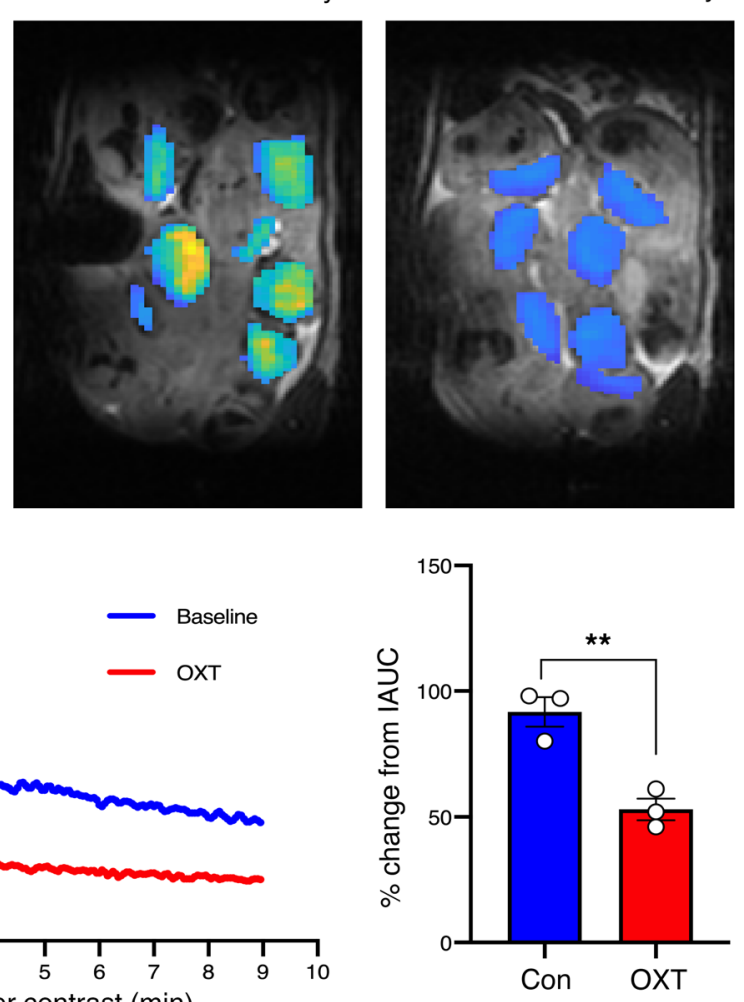

200

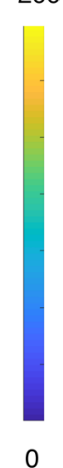

0

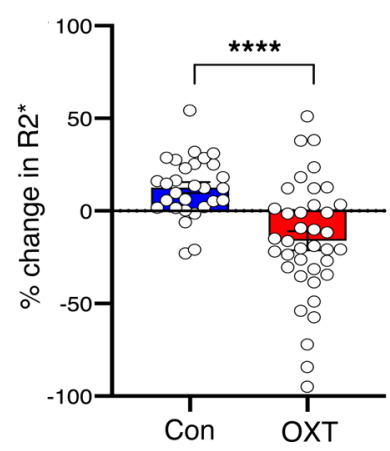

Figure 1. Placental perfusion is significantly impaired after OXT-induced aberrant uterine contractility. (A) Middle and right show the initial area under the curve (IAUC) from the baseline and post-OXT dynamic contrast-enhanced MRI experiments overlaid onto the corresponding anatomical T2-weighted images from 1 slice shown on the left. (B) Left: Placental relative signal enhancement after Dotarem injection is significantly decreased after OXT compared with baseline. Right: Comparison of the changes in IAUC (averaged across all placentas) from baseline after either saline or OXT showing an approximately $45 \%$ decrease after OXT, confirming that OXT-induced uterine hypercontractility was associated with significantly reduced uteroplacental perfusion ( $n=3$ per group). Data were analyzed with 2-tailed Student's $t$ test and presented as mean \pm SEM; ${ }^{* *} P<0.01$. (C) OXT-induced hypercontractility significantly decreases placental R2*. Left: Illustrative fast spin echo (FSE) images from an OXT-treated dam showing placental regions of interest (ROIs). Right: Placental R2* was measured in 29 and 38 placentas from saline-treated $(n=3)$ or OXT-treated $(n=6)$ dams, respectively. OXT treatment decreased placental R2* by approximately $40 \%$, suggesting either severe uteroplacental perfusion or profound placental squeeze draining all blood, including deoxygenated blood, away from the placenta. Data were analyzed with Mann-Whitney $U$ test and presented as mean $\pm \mathrm{SEM}$; ${ }^{* * *} P<0.0001$.

OXT-induced uterine hypercontractility is associated with social behavioral abnormalities in the adolescent male offspring. Based on the effects of OXT-induced aberrant uterine contractility on the fetal brain, we predicted long-term functional consequences for the offspring. Therefore, in a separate set of experiments, we evaluated OXT- and saline-exposed (CON-exposed) offspring on several behavioral tests designed to assess locomotor 
A
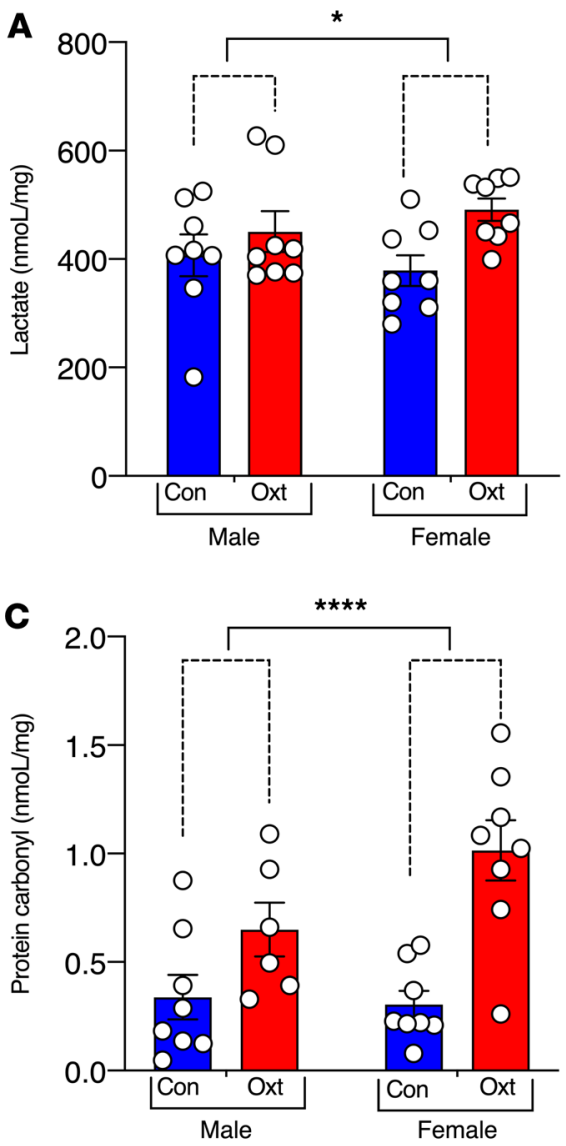

B
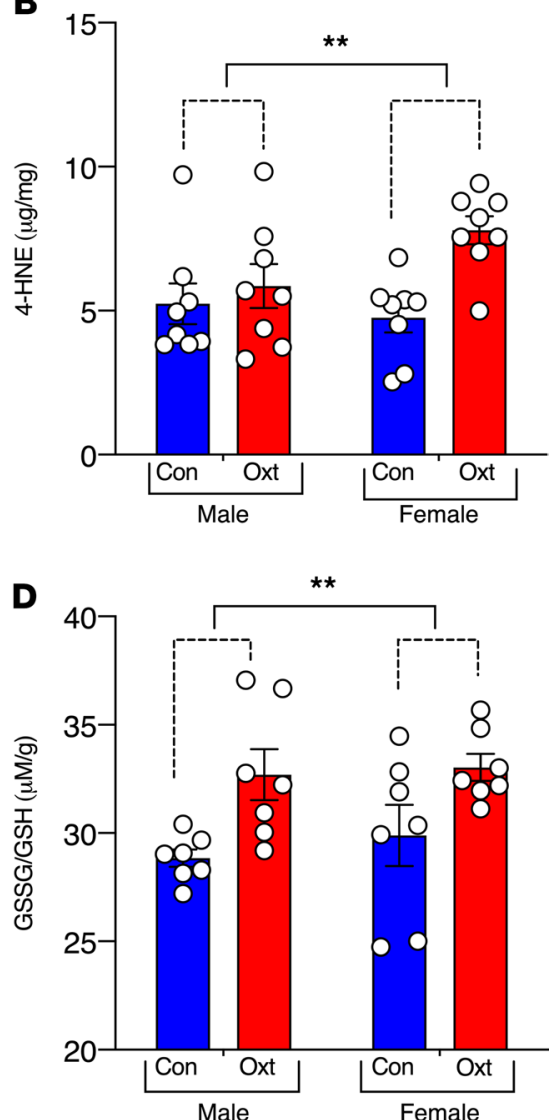

Figure 2. Transient OXT-induced uterine hypercontractility causes oxidative stress in the fetal brain. In utero exposure to OXT-induced uterine hypercontractility increased the concentration of lactate (A), 4-hydroxynonenal (B), protein carbonyl (C), and oxidized glutathione (D) in the fetal brain, strongly suggesting the presence of oxidative stress $(n=8$ male and female pups from 8 dams per treatment condition). Data were analyzed with 2-way ANOVA and presented as mean $\pm \mathrm{SEM} ;{ }^{*} P<0.05,{ }^{* *} P<0.01$, and ${ }^{* * *} P<0.0001$

activity, anxiety-related measures, social memory, and associative learning and memory between P28 and P45 (Figure 4, A-D). There were no significant treatment- or sex-related differences in general locomotor activity (1-hour open-field test; Figure 4A) and anxiety-related measures (elevated plus maze; Figure 4B). However, social investigation time of a novel conspecific was significantly decreased in male, but not female, OXT-exposed offspring relative to male CON-exposed offspring (Figure 4C). In the observational fear learning (OFL) test (Figure 4D), OXT-exposed offspring exhibited significantly increased freezing levels during the last $2 \mathrm{~min}$ utes of the training period despite comparable levels during baseline and the first 2 minutes of training, but no sex effects were observed. However, no differences between groups were observed for the OFL contextual fear test conducted 24 hours later. The differences in freezing levels between the groups during training were not likely due to differences in ambulatory activity because they performed similarly on this variable during a 1-hour open-field test. In a separate set of experiments, we assessed whether these behavioral changes were accompanied by differential expression of $N r x n 1$, known to be associated with social behavior, in the amygdala, somatosensory cortex, and anterior cingulate cortex of P28 offspring. We detected a significant decrease in the expression of Nrxn1 in the amygdala of both male and female OXT-exposed P28 offspring, while these changes were not observed in the anterior cingulate and the somatosensory cortex (Supplemental Figure 3). Collectively, these results suggested that the main neurobehavioral effects of exposure to OXT-induced hypercontractility in utero are a male-specific impairment of social investigation accompanied by brain regionspecific changes in the expression of genes related to social and emotional behaviors.

OXT-induced uterine hypercontractility causes persistent sex-specific increase in proteins from the mitochondrial electron transport chain. Because of sex-dependent changes in behavior, we investigated gene expression changes related to oxidative stress 1 hour after OXT-induced uterine hypercontractility in both male and 
A

Top 25 FDR significant GO biological processes bar plot

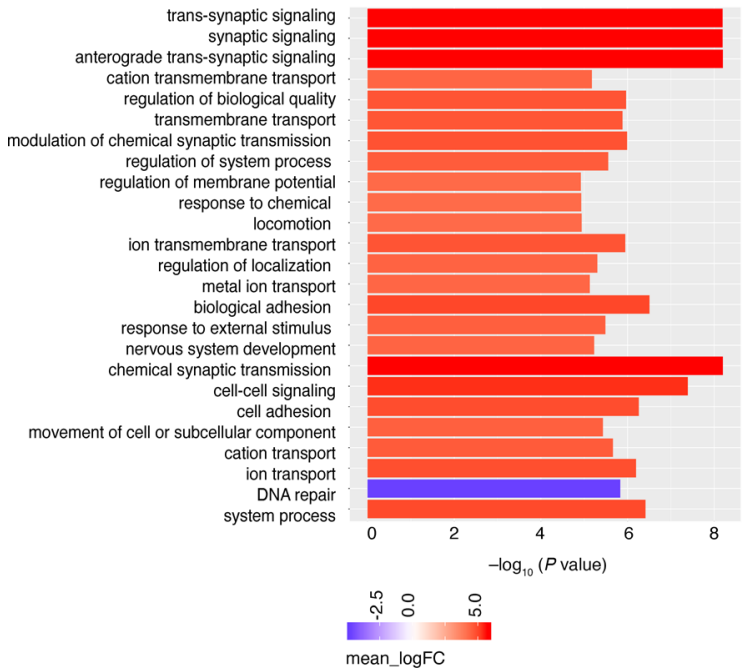

C

Top 25 upregulated $P$ value significant KEGG bar plot

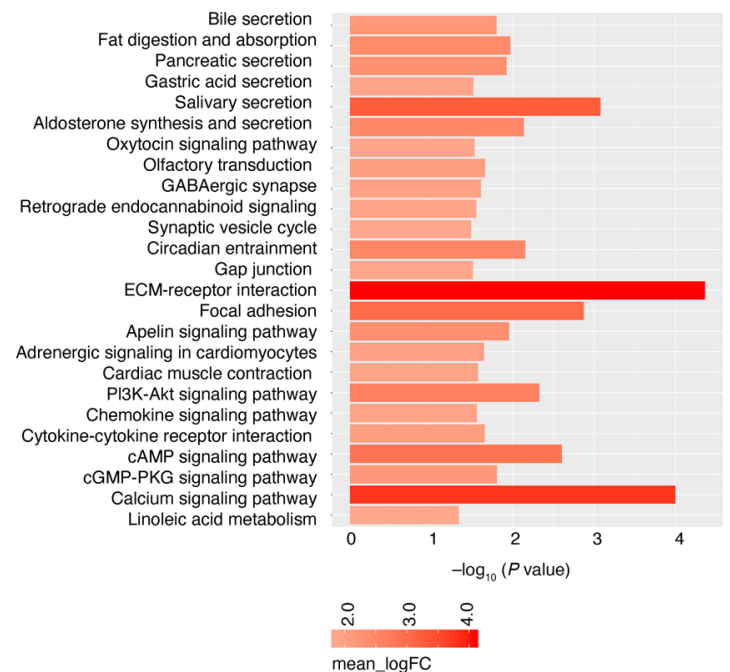

B

Top 25 FDR significant GO molecular functions bar plot

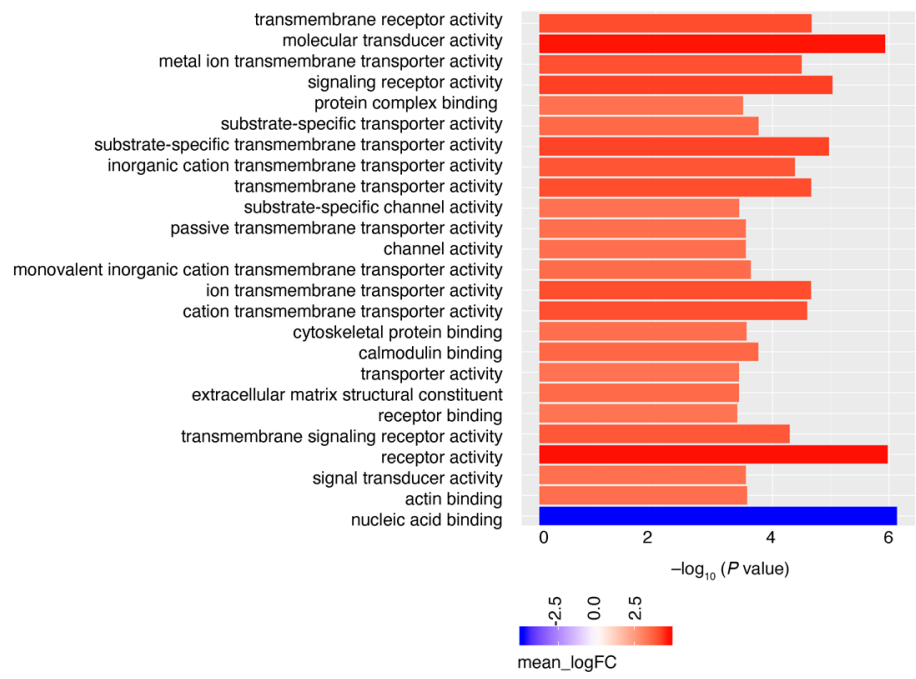

Top 25 downregulated $P$ value significant KEGG bar plot

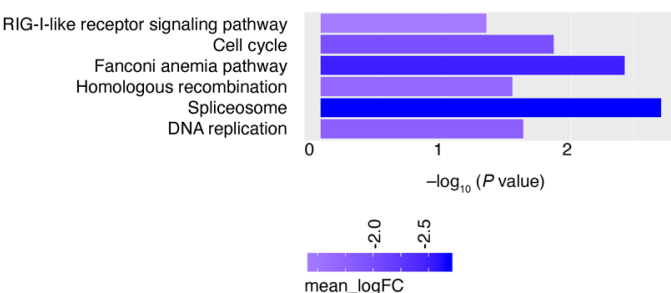

Figure 3. Fetal cortical transcriptomic changes induced by OXT-induced uterine hypercontractility. Top 25 significantly up- and downregulated genes for Gene Ontology (GO) biological processes (A) and molecular functions (B) after OXT-induced hypercontractility. (C) Significantly upregulated genes and (D) significantly downregulated genes with Kyoto Encyclopedia of Genes and Genomes (KEGG) analysis ( $n=5$ pups per group with each pup from a unique dam; $n=5$ dams each for saline and OXT).

female offspring. Specifically, we performed planned comparisons of treatment-related differences in the expression of select target genes related to electron transport chain/oxidative stress (ETC/oxidative stress), antioxidant defense, and hypoxia/apoptosis pathways using prevalidated TaqMan-specific probes. We attempted to perform this in the anterior cingulate cortex and amygdala, but quick liquefaction of the E21 pup brain prevented us from accurate dissection of these brain regions. Therefore, the entire fetal cortex was assessed. Multiple genes were differentially expressed in a sex-dependent manner; overall, there was an increased expression of genes related to ETC/oxidative stress ( $m t-N d 4, m t-N d 5, m t-A t p 8)$, antioxidant defense (Sod2, Cygb), and hypoxia/apoptosis (Casp8, Hifla) pathways in treated males versus females (Figure 5A). Oxtr expression was not significantly different with OXT treatment, nor was there a sex/OXT interaction at this time point (Supplemental Figure 4). We then selectively quantified changes in proteins emblematic of these pathways with immunoblots. HIF1A and CASP3 were assessed in nuclear and whole cortical lysates, respectively, at 1 hour and 18 hours. There were no treatment- or sex-related differences in 
A

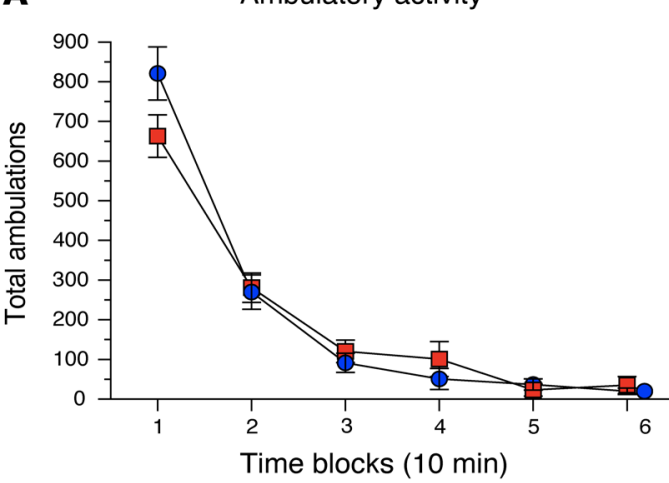

B

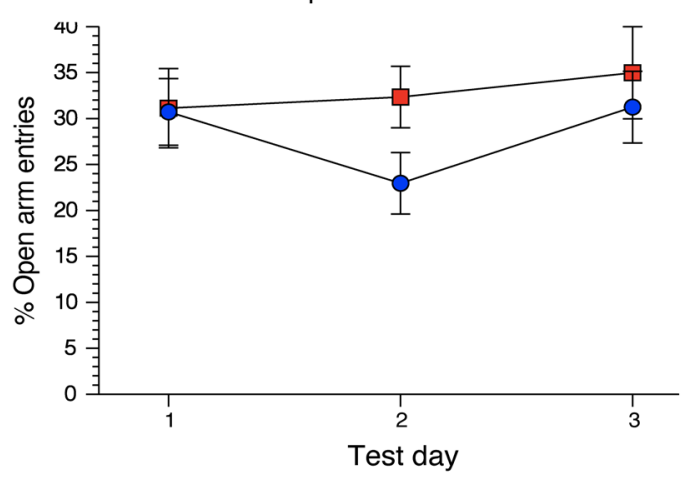

C

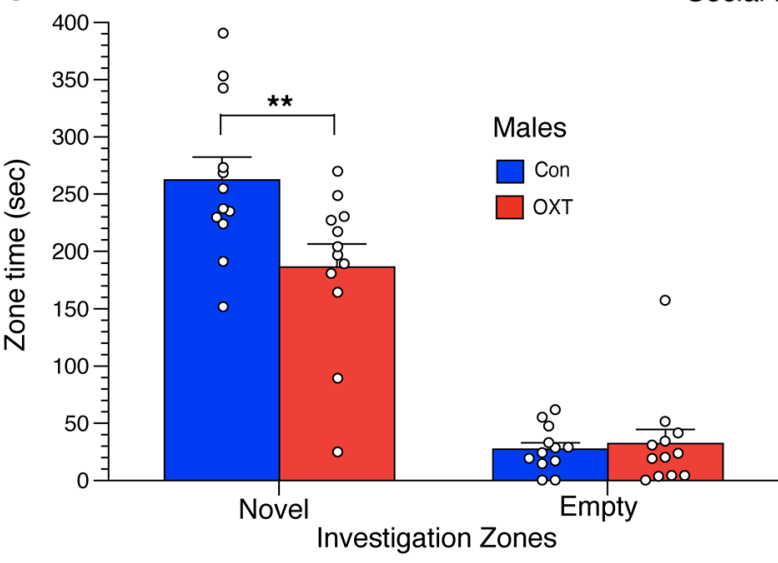

D OFL Day 1: Baseline/Training

Social approach

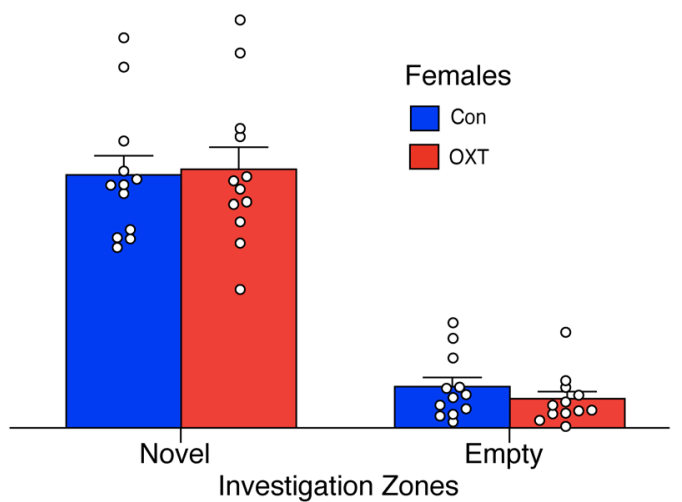

OFL Day 2: Contextual Fear
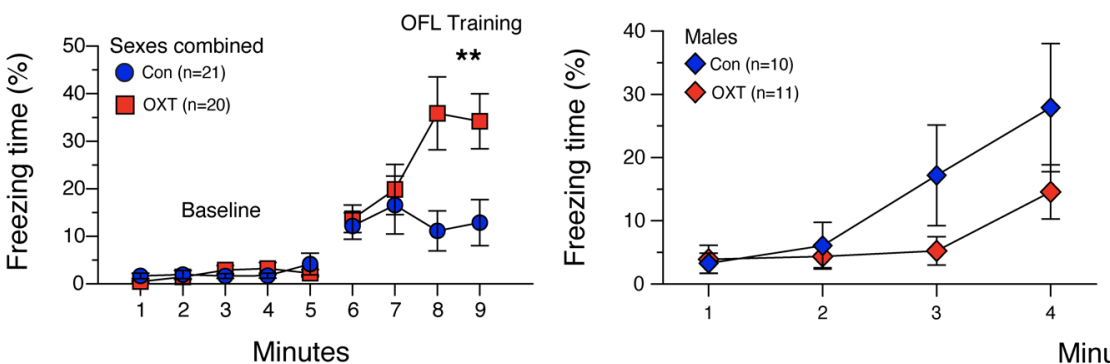

Females

$\Delta \operatorname{Con}(n=11)$

$\triangle \operatorname{OXT}(\mathrm{n}=9)$

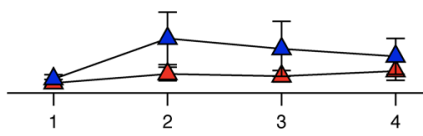

Minutes

Figure 4. OXT-induced uterine hypercontractility is associated with male-specific decrease in social investigation and an increase in empathy-like fear. There were no differences in ambulatory activity in the open-field test (A) and the percentage of open arm entries in the elevated plus maze (B) between the CON and OXT groups. (C) However, male OXT-exposed offspring spent significantly less time investigating a novel rat compared with CON male offspring. No differences were observed between CON and OXT female offspring. (D) OXT offspring showed significantly increased freezing levels in the OFL test during the last 2 minutes of training, suggesting an enhanced empathy-like response. However, there were no differences in the contextual fear test 24 hours later ( $n=12$ male and female offspring from 9 independent litters per treatment condition with exclusions as reported in supplemental materials). Data analyzed with repeated-measures ANOVA (followed by Bonferroni's correction if required) and presented as mean $\pm S E M$; ${ }^{* *} P<0.01$.

either HIF1A or CASP3 (Supplemental Figure 5), suggesting that the related gene expression changes were probably transient. Because of the predominance of mitochondrially coded genes among genes that were differentially expressed at 24 hours after OXT-induced hypercontractility, we focused on oxidative phosphorylation (OXPHOS) proteins in the anterior cingulate cortex, considering the importance of this brain region for social behavior and cognition (20-25). OXPHOS proteins were quantified in the mitochondrial fraction isolated from the anterior cingulate cortex of male and female P28 offspring. We observed that ETC complex I, III, and IV proteins were selectively higher in the anterior cingulate cortex of P28 male OXT-exposed offspring (Figure 5B), suggesting that transient but severe hypoxic events in utero can cause persistent mitochondrial changes. 
A

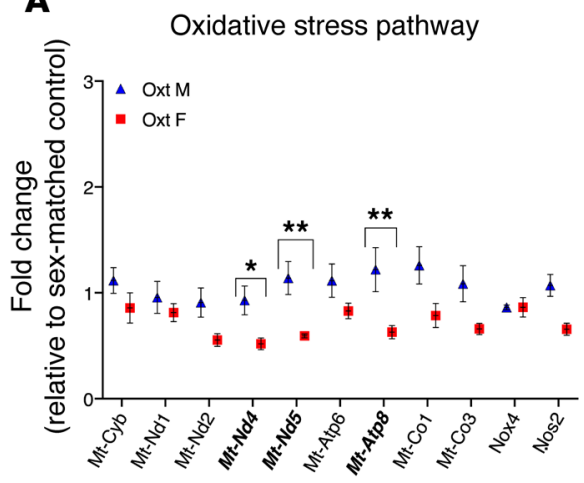

Antioxidant genes

Hypoxia/apoptosis pathway

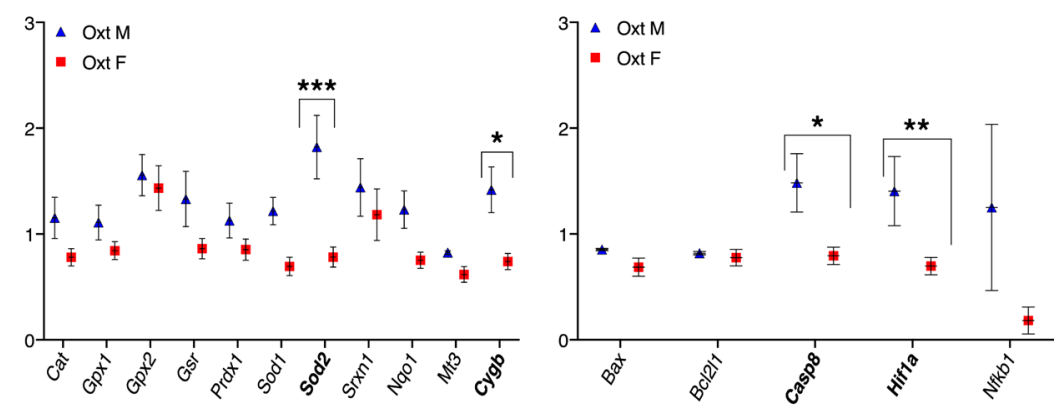

B
ETC Complex I

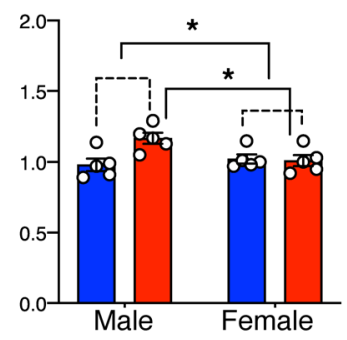

ETC Complex IV

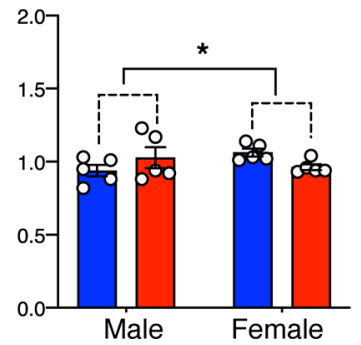

ETC Complex III

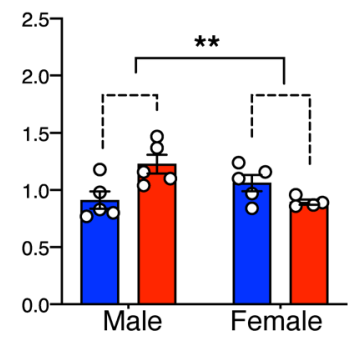

Comp II (30 kD)

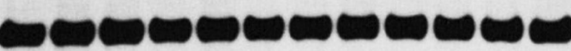

Comp I (20 kD)

$\operatorname{VDAC}(55 \mathrm{kD})$
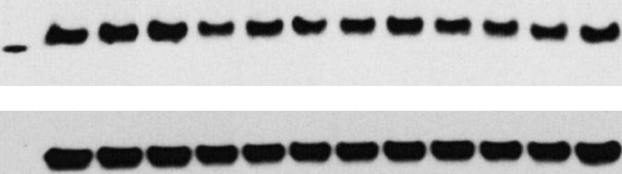

Figure 5. OXT-induced hypercontractility causes sex-dependent gene expression changes and enduring upregulation of OXPHOS proteins. (A) OXT-induced hypercontractility causes sex-dependent gene expression changes in the E21 fetal cerebral cortex. Multiple genes related to ETC/oxidative stress (mt-Nd4, $m t-N d 5, m t-A t p 8)$, antioxidant defense (Sod2, Cygb), and hypoxia/apoptosis (Casp8, Hif1a) pathways were differentially expressed in a sex-dependent manner 1 hour after OXT-induced hypercontractility. Data were analyzed with 2-way ANOVA followed by Holm-Šidák multiple-comparisons test and presented as mean $\pm \mathrm{SEM}$ ( $n=6$ per sex per treatment condition); ${ }^{*} P<0.05,{ }^{* *} P<0.01$, and ${ }^{* * *} P<0.001$. (B) OXT-induced hypercontractility causes enduring upregulation of OXPHOS proteins in the anterior cingulate cortex of male P28 offspring. Left: Representative immunoblots of anterior cingulate cortex homogenates from P28 offspring showing increased expression of proteins related to the mitochondrial ETC (OXPHOS) in male offspring. Right: Scatter plots show a significant treatment/sex interaction for complex I $([F(1,16)=6.5])$, complex III $([F(1,15)=12.0])$, and complex IV $([F(1,16)=4.6])$. Normalization was done with mitochondrial VDAC1 protein. Data were analyzed with 2-way ANOVA and presented as mean \pm SEM $\left(n=5\right.$ per sex per treatment condition); ${ }^{*} P<0.05$, and ${ }^{* *} P<0.01$.

OXT-exposed male offspring demonstrate enduring impairment in cortical functional connectivity. Complex behavioral changes cannot be explained by differences in gene and protein expression alone. Therefore, we examined the effects of OXT exposure on systems-level brain organization using functional connectivity optical intrinsic signal imaging (fcOISI) (26) in male offspring exposed to either OXT or saline. Candidate ROIs were placed in cortical areas corresponding to cingulate, motor, somatosensory, retrosplenial, and visual networks (Figure 6A). Saline-treated rats exhibited robust patterns of functional connectivity that feature prominently in healthy rats. These characteristics included strong positive (reds) ipsilateral correlation adjacent to each ROI, as well as mirrored homotopic functional connectivity contralateral to each ROI (Figure 6B). Additionally, strong anticorrelations (blues) were present between opposed functional networks, e.g., among sensorimotor and retrosplenial cortices. These features were altered in the OXT-treated group. For example, correlation difference maps reveal substantially reduced homotopic functional connectivity strength in cingulate cortex (Figure 6C), along with large changes in ipsilateral connectivity within cingulate, visual, and retrosplenial regions (reds, Figure 6C). The magnitude of anticorrelations between sensorimotor and visual and parietal regions, as well as between retrosplenial and lateral sensory regions, 
was also attenuated (blues, Figure 6C). One of the most salient differences between saline- and OXT-treated rats was the spatial extent of positive correlations exhibited by a given ROI. To quantify this metric, we constructed maps of global node degree, calculated as the number of cortical functional connections exhibited by a given pixel having a correlation strength higher than $Z(r)>0.3$ (Figure 6D). Saline-treated animals exhibited high connection number (yellows, whites) in retrosplenial, visual, and motor regions. OXT-treated rats exhibited more global reductions in node degree, as indicated by the darker shades of red and black over large portions of the cerebral cortex. Marked regional differences in this measure were observed in portions of motor, somatosensory, parietal, cingulate, and visual cortices (Figure 6D). Taken together, exposure to OXT-induced uterine hypercontractility in utero affects functional brain organization within and across functionally distinct brain systems.

\section{Discussion}

In this translational study with term pregnant rats, we show that OXT-induced aberrant uterine contractility was associated with severe but transient impairment of uteroplacental perfusion with significant functional consequences for the offspring. Specifically, we observed significant oxidative stress in the fetal brain, with an increase in both biomarkers and genes mediating oxidative stress. These changes were accompanied by social behavioral abnormalities especially in males, significant reductions in functional connectivity between different brain regions, and an enduring upregulation of mitochondrial ETC complexes in the anterior cingulate cortex. Collectively, our findings suggest that even transient but severe impairment of placental perfusion can have lasting detrimental consequences for the offspring.

Our studies appear to be the first to comprehensively examine the impact of transient but severe intrapartum hypoxemia resulting from aberrant uterine contractility on the fetal brain. Our model is more pragmatic in that it avoids complex vascular surgery to reduce uteroplacental perfusion, but more importantly, allows for quick reversibility of the insult to better reflect complications during human labor (13, 14, 27-29). Our findings challenge the prevailing assumption that neonatal metabolic status is a good surrogate for the well-being of the fetal brain and suggests that even a short-duration hypoxic insult could be damaging to the fetal brain. Furthermore, our findings offer mechanistic evidence linking intrapartum hypoxia, oxidative stress, and persistent abnormalities in functional connectivity as the possible drivers of neurodevelopmental disorders observed after acute obstetric complications (1-5, 30-34).

Disruption to the OXT/OXTR system could play a role in the observed biochemical and behavioral abnormalities. We minimized this possibility by choosing a dose of OXT that was not associated with significant placental transfer in our model (35). Though we cannot definitively rule out activation of transient OXTR signaling at the time of OXT injection, multiple lines of evidence from our studies suggested minimal involvement of the OXT/OXTR system (RNA-Seq data, TaqMan quantitative PCR [qPCR]) at a later time point (Supplemental Figure 2). This may appear to be at odds with the study by Kenkel et al. showing acute effects of OXT on the fetus (36), but we posit possibilities why our outcomes were different: (a) we administered OXT intravenously, causing a profound and immediate tetanic uterine contraction, compared with an i.p. injection in the Kenkel study, and (b) the associated drastic reduction in uteroplacental perfusion most likely decreased the delivery of OXT-containing maternal blood to the placenta and to the fetus. Our study is perhaps more comparable to the study by Boksa et al. demonstrating that acute administration of OXT before birth worsened anoxic brain injury in the neonatal rat brain (37). With this background, we believe that the observed effects in the developing brain are more likely due to indirect effects from the tetanic uterine contraction rather than the direct effects of OXT.

Observation of severe reduction in uteroplacental perfusion after OXT-induced uterine contractility motivated us to explore hypoxic stress in the fetal brain as a possibility. This line of investigation was reinforced by evidence for increased fetal brain lactate and biomarkers of oxidative stress after OXT. Though examination of the global fetal cortical transcriptomic changes 24 hours after OXT did not reveal involvement of specific hypoxia-associated pathways, there was a preponderant differential expression of genes from the mitochondrial ETC. Taken together with the central role of brain mitochondria in responding to hypoxic and oxidative stress (38-41), and the brain region-specific persistent alteration of mitochondrial ETC proteins in juvenile offspring, we determined that one of the major effects of OXT-induced uteroplacental ischemia was oxidative stress with possible mitochondrial dysfunction.

A major finding of our behavioral studies was an impairment of social investigation in male offspring exposed to OXT-induced hypercontractility in utero. We pursued this with evaluation of the expression of 
A

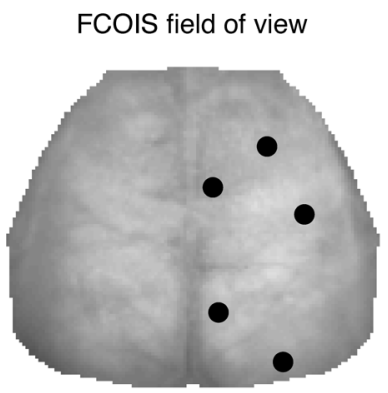

C

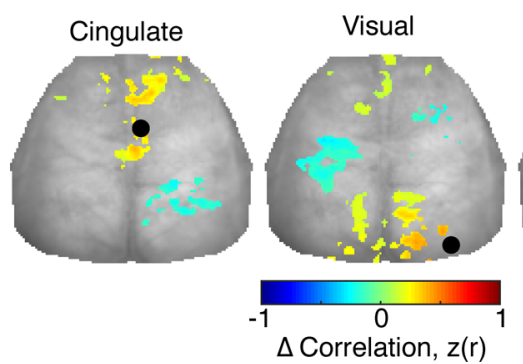

B

Saline

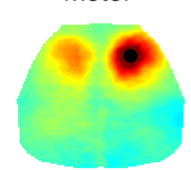

OXT
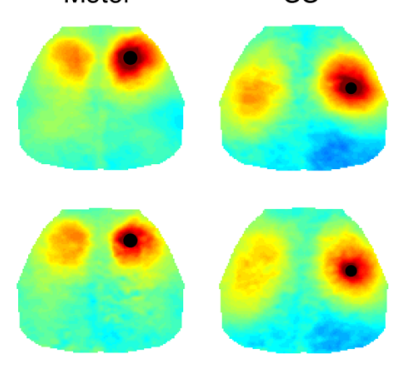

Cing
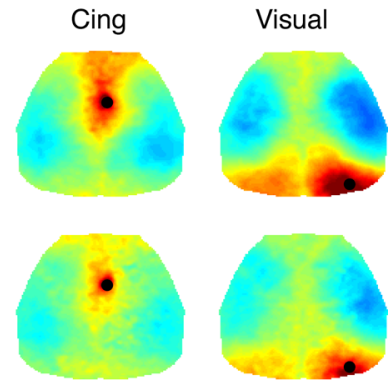

D

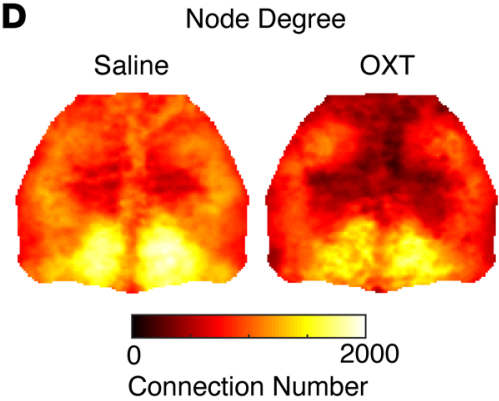

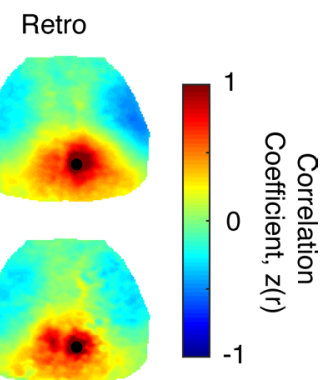

Saline minus OXT

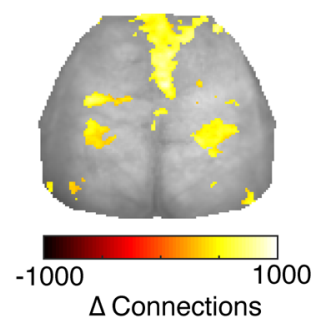

Figure 6. In utero OXT exposure affects functional brain organization in juvenile male offspring. fcOISI imaging was performed in P23 male offspring exposed to either saline or OXT in utero. (A) fcOIS field of view. Seeds for functional connectivity analysis (black dots on representative white light image of the rat skull) were placed in cortical areas corresponding to cingulate, motor, somatosensory (SS), retrosplenial, and visual networks. (B) Top row: Salinetreated rats exhibited patterns of functional connectivity with strong positive (reds) ipsilateral correlation adjacent to and contralateral from each seed and strong anticorrelations (blues) between opposed functional networks (e.g., between sensorimotor and retrosplenial cortices). Qualitatively, these general features of healthy patterns of functional connectivity were reduced in OXT-treated rats (bottom row). (C) Functional connectivity difference maps in select networks. Substantial reduction in ipsilateral connectivity was observed in cingulate, visual, and retrosplenial regions. Anticorrelated activity (blues) was also significantly reduced between somatomotor areas and visual regions, along with lateral sensory and retrosplenial regions. (D) Maps of node degree for each group were calculated by thresholding each pixel's functional connectivity map at $Z(r)>0.3$ and counting all pixels above this threshold. Salinetreated animals exhibited high connection number (yellows, whites) in retrosplenial, visual, and motor regions. OXT-treated rats exhibited more global reductions in node degree. Marked regional differences in node degree were observed in portions of motor, somatosensory, parietal, cingulate, and visual cortices (difference map). Significance determined by 2-tailed Student's $t$ test assuming unequal group variance at each pixel and corrected for multiple comparisons using FDR correction on a cluster-wise basis ( $n=7$ saline and 5 OXT).

Nrxn1, previously associated with social behavior, in brain regions known to be integral to the neurocircuitry of social interaction (amygdala, anterior cingulate cortex, somatosensory cortex) (42-49). Expression of Nrxn1 was differentially regulated in the brain regions of OXT-exposed offspring regardless of the sex of the offspring; Nrxn1 was markedly downregulated in the amygdala but unchanged in the anterior cingulate and the somatosensory cortices of OXT versus control offspring. Because these results do not explain male-specific impairment of social behavior, we speculate that these changes could be due to sex differences in the response to oxidative stress $(38,50-54)$. The considerable sex difference in the expression of genes mediating oxidative stress 1 hour after OXT and the presence of persistently altered mitochondrial ETC proteins in the anterior cingulate cortex of only P28 male offspring support this viewpoint.

Mitochondrial dysfunction has also been recently demonstrated to lead to significant reductions in longrange axonal connectivity (55). Because structural connectivity is a required substrate for functional connectivity, OXT-induced changes in factors affecting anatomical connections between neurons could affect functional brain network organization (i.e., segregation and integration). Our general finding of impaired cortical functional connectivity in male offspring treated with OXT is broadly supportive of this notion. More specifically, OXT-treated rats exhibited significant reductions in the magnitude of anticorrelations between functionally distinct networks positioned across the anterior-posterior axis. In humans, observations of anticorrelated spontaneous brain activity resulted in some of the first accounts of intrinsic brain organization (56). Dynamic patterns of anticorrelated activity segregate distinct processes across networks during both task and resting state paradigms $(56,57)$, with the most common example in humans occurring between the dorsal attention network and default mode network (58). Altered synchrony across large cortical distances, as observed by reduced anticorrelated activity, could prevent internetwork communication necessary for higher-order integration. Another marked distinction between saline- and OXT-treated rats was significantly 
altered ipsilateral node degree in portions of motor, somatosensory, parietal, cingulate, and visual cortices. These reductions could be indicative of network-level inefficiencies important for segregation. For example, in mice we have recently shown (59) that improved tactile proprioception after stroke was associated with increased node degree in motor, somatosensory, and parietal cortices - all regions relevant for processing proprioception and touch (60). In the present study, reduced within-network connectivity (i.e., node degree) combined with poorer integration across networks (i.e., reduced anticorrelations) could impair multidomain behavioral output, as demonstrated by poorer social and emotional performance of the OXT-treated group.

Our findings have to be interpreted in the context of a few limitations. First, it is not possible to extrapolate the duration of placental ischemia-hypoxia in rats to humans. Furthermore, human babies are likely to be delivered if hypoxia persists long enough to cause concerning fetal heart rate changes. Nevertheless, the developing human brain is generally more sensitive to hypoxia than the rodent brain and is more likely to be susceptible to mild hypoxic injury (61). Second, we did not treat placental ischemia-hypoxia with oxygen administration in the dam, a practice that is common during human labor. Therefore, there is a possibility that oxidative stress could be worsened in this setting. Third, we focused only on 3 brain regions based on behavioral changes, but it is likely that we may have missed important changes elsewhere. Similarly, a brain region-specific analysis of oxidative stress immediately after OXT was not possible because easy liquefaction of the fetal brain prevented an accurate dissection of brain regions. Despite these limitations, our findings provide detailed insights into the possible mechanisms by which transient placental ischemia-hypoxia could induce persistent dysfunction in the developing brain.

In conclusion, transient intrauterine ischemia-hypoxemia was associated with male-specific differences in social behavior, differential expression of selective oxidative stress genes in the developing cortex, and enduring upregulation of mitochondrial ETC proteins in the anterior cingulate cortex of male offspring. Collectively, our findings support the idea that severe but reversible hypoxic stress could induce permanent dysfunction in the developing brain and raise the possibility of persistent mitochondrial dysfunction as the link between transient intrauterine asphyxia and neurodevelopmental disorders.

\section{Methods}

Animals and drugs. Timed pregnant Sprague-Dawley rats (Charles River Laboratories) were used for all experiments. OXT (Selleck Chemicals) was prepared as a $1 \mathrm{mg} / \mathrm{mL}$ solution in sterile normal saline and diluted as necessary. We performed a pilot study with 3 doses of OXT $(10 \mu \mathrm{g} / \mathrm{kg}, 100 \mu \mathrm{g} / \mathrm{kg}$, and $1 \mathrm{mg} / \mathrm{kg})$ to determine the duration of externally visible uterine contraction and confirmed that $100 \mu \mathrm{g} / \mathrm{kg}$ caused a predictable 10 - to 15 -minute tetanic uterine contraction. Uterine contraction induced by $10 \mu \mathrm{g} / \mathrm{kg}$, however, was variable in length, while that induced by $1 \mathrm{mg} / \mathrm{kg}$ was so severe that it occasionally resulted in stillborn pups. Furthermore, $100 \mu \mathrm{g} / \mathrm{kg}$ OXT administered to the mother was not accompanied by an increase in fetal brain OXT in our previous study (35), suggesting a lack of placental transfer at this dose. During the course of these experiments, we also developed a rapid method to identify the sex of the pups using visual inspection of the anogenital distance at E21. Another experimenter cross-checked and verified the sex by performing a minilaparotomy to identify the presence (male) or absence (female) of testes and seminal vesicles. The total number of animals used for specific experiments is detailed in Supplemental Table 1. Technical details are outlined in Supplemental Tables 2 and 3. Litter data and weight gain trajectories of the offspring are presented in Supplemental Figure 6.

Placental imaging. For the dynamic contrast-enhanced (DCE) MRI scans, Sprague-Dawley dams at E21 $(n=3)$ were anesthetized with isoflurane in $100 \%$ oxygen and imaged on an Agilent 4.7T DirectDrive MRI system using a 7.2-cm-inner diameter quadrature radiofrequency coil. Anatomic scans were initially acquired across the entire abdomen using a respiratory-gated $\mathrm{T} 2$-weighted $2 \mathrm{D}$ multislice fast spin echo sequence $\left(0.5 \times 1 \times 1 \mathrm{~mm}^{3}\right.$ resolution, zero-padded to $0.5 \times 0.5 \times 1 \mathrm{~mm}^{3}$; repetition time/echo time [TR/ $\mathrm{TE}]=2000 / 48 \mathrm{~ms}$; echo train length $=4 ; 4$ averages; respiratory gated; imaging time approximately 6 minutes depending upon respiration rate). Placental blood flow was measured by DCE MRI with a tail vein injection of Dotarem (Guerbet, $0.2 \mathrm{mmol} / \mathrm{kg}$ ). The rats were then injected with $100 \mu \mathrm{g} / \mathrm{kg}$ of OXT through a $22 \mathrm{G}$ tail vein catheter to induce tetanic uterine contraction. Following the cessation of gross abdominal motion, typically around 30 minutes as assessed by nongated low-resolution scouts, the anatomic scan was reacquired to account for placental displacement during the contraction period, and the DCE measurement was repeated with a repeat dose of the same contrast agent. Placental perfusion was quantified as the IAUC of the DCE time intensity for each placental voxel using the DCE@urLAB package (62). Each rat placenta 
was manually segmented from pre- and post-OXT anatomical images using customized MATLAB routines and interpolated onto the IAUC maps. Because the DCE protocol involved 2 injections of contrast agent separated by approximately 50 minutes, the time under anesthesia and the presence of residual contrast agent from the first injection could potentially distort the post-OXT DCE results. To examine the size of these effects, we repeated the DCE protocol where we injected the same volume of saline instead of OXT $(n=3)$ followed by comparison of the percentage change in IAUC between the saline and OXT groups. Placental R2* mapping was performed using a 3D multiecho gradient echo sequence ( 0.75 -mm isotropic resolution; $\mathrm{TR} / \mathrm{TE} / \Delta \mathrm{TE}=40 / 1.7 / 4 \mathrm{~ms} ; 6$ echoes; flip angle $=15$ degrees, 4 minutes, 16 seconds) to measure changes in deoxyhemoglobin concentration after $100 \mu \mathrm{g} / \mathrm{kg}$ of OXT $(n=6)$ or an equivalent volume of saline $(n=3)$ in a separate set of experiments. Rat placentas were manually segmented from pre- and post-OXT images using customized MATLAB routines, and optimal estimates of R2* were determined for each placenta using software implementing Bayesian probability theory. For the saline-injected rats, a 30-minute delay was observed before acquiring postinjection scans.

Lactate assay. To determine whether OXT-induced uterine contractions induce hypoxemia in the fetal brain, we assayed for fetal brain lactate 4 hours after $100 \mu \mathrm{g} / \mathrm{kg}$ i.v. OXT or saline in timed pregnant E21 Sprague-Dawley dams ( $n=8$ per group). Briefly, whole male and female fetal cerebral cortices were homogenized on ice and deproteinized with a $10-\mathrm{kDa}$-molecular weight cutoff spin filter to remove lactate dehydrogenase. Protein concentrations were determined using BCA Protein Assay Kit (Thermo Fisher Scientific), followed by lactate assay (Lactate Colorimetric Assay Kit II, MilliporeSigma) according to manufacturers' instructions. Results calculated from the standard curve were expressed as nmol/mg brain protein.

Oxidative stress assays. To determine whether OXT causes oxidative stress in the fetal brain, we assayed male and female fetal brains for 4-hydroxynonenal (4-HNE, a by-product of lipid peroxidation), protein carbonyl, and the antioxidant GSSG/GSH from the same E21 dams used for the lactate assay. Briefly, whole fetal cerebral cortices from male and female pups were homogenized in PBS buffer on ice. Protein concentrations were determined using BCA Protein Assay Kit (Thermo Fisher Scientific), followed by 4-HNE assay (OxiSelect HNE Adduct Competitive ELISA Kit), protein carbonyl (OxiSelect Protein Carbonyl ELISA Kit), and total glutathione assay (OxiSelect GSSG/GSH Assay Kit) according to manufacturers' instructions. All assays were supplied by Cell Biolabs, Inc. Results calculated from the standard curve were expressed as $\mu \mathrm{g} / \mathrm{mg}$ of brain protein.

$R N A$-Seq and analysis. E21 timed pregnant SD rats were treated with either $100 \mu \mathrm{g} / \mathrm{kg}$ OXT or saline through a $22 \mathrm{G}$ tail vein catheter $(n=5 \mathrm{each})$. Twenty-four hours later, fetal cerebral cortices were harvested under isoflurane anesthesia. Total RNA was extracted from the right cerebral cortex using an RNeasy kit (QIAGEN). Only RNA with RNA integrity number greater than 9.5 was used for RNA-Seq. Samples were prepared according to the library kit manufacturer's protocol, indexed, pooled, and sequenced on an Illumina HiSeq. Base calls and demultiplexing were performed with Illumina's bcl2fastq software and a custom Python demultiplexing program with a maximum of 1 mismatch in the indexing read. RNA-Seq reads were then aligned to the Rattus norvegicus Ensembl Rnor_5.0 top-level assembly with STAR version 2.0.4b. Gene counts were derived from the number of uniquely aligned unambiguous reads by Subread: featureCount version 1.4.5. Isoform expression of known Ensembl transcripts was estimated with Sailfish version 0.6.13. Sequencing performance was assessed for the total number of aligned reads, total number of uniquely aligned reads, and features detected. The ribosomal fraction, known junction saturation, and read distribution over known gene models were quantified with $\mathrm{RSeQC}$ version 2.3. All gene counts were then imported into the R/Bioconductor package EdgeR, and trimmed mean of M values (TMM) normalization size factors were calculated to adjust for samples with differences in library size. Ribosomal genes and genes not expressed in at least 4 samples greater than $1 \mathrm{count} / \mathrm{million}$ were excluded from further analysis. The TMM size factors and the matrix of counts were then imported into the R/Bioconductor package limma. Unknown latent effects were then estimated with the R/Bioconductor package SVA. Weighted likelihoods based on the observed mean/variance relationship of every gene and sample were then calculated for all samples with voomWithQualityWeights. The performance of all genes was assessed with plots of the residual standard deviation of every gene to their average log count with a robustly fitted trend line of the residuals. Differential expression analysis was then performed to analyze for differences between conditions, and the results were filtered for only those genes with Benjamini-Hochberg FDR-adjusted $P$ values less than or equal to 0.05 . For each contrast extracted with limma, global perturbations in known KEGG pathways were detected using the R/Bioconductor package GAGE9 to test for changes in expression of the reported $\log _{2}$ fold changes reported by limma in each term versus the background $\log _{2}$ fold changes of all genes found 
outside the respective term. The R/Bioconductor package Pathview10 was used to display heatmaps or annotated KEGG graphs across groups of samples for each KEGG pathway with a $P$ value less than or equal to 0.05. RNA-Seq data have been deposited in the National Center for Biotechnology Information's Gene Expression Omnibus and are accessible through GEO Series accession number GSE146155 (https:// www.ncbi.nlm.nih.gov/geo/query/acc.cgi?acc=GSE146155).

Behavioral experiments. Male and female offspring of E21 Sprague-Dawley dams ( $n=9$ each) treated with OXT $(100 \mu \mathrm{g} / \mathrm{kg})$ or saline (OXT or CON, respectively) were subjected to behavioral testing. There were no differences in litter size, sex ratio, or survival of the offspring from both treatment groups. Pups were weaned on P21 and subsequently evaluated on a battery of behavioral tests from postnatal day P26 through P45 in the following order: (a) 1-hour open field, (b) social approach, (c) elevated plus maze, and (d) OFL. The OXT and CON rats were tested in 2 cohorts ( $n=6$ and 3 dams each, respectively), for which identical behavioral protocols were used. A total of 12 male and female offspring from the OXT and CON group were tested. Details of all behavioral experimental procedures were described previously (63) and are included in the Supplemental Methods.

TaqMan $q P C R$. Male and female fetal brains exposed to either $100 \mu \mathrm{g} / \mathrm{kg}$ OXT or saline $(n=8$ per group) in utero were harvested and immediately stored at $-80^{\circ} \mathrm{C}$. RNA was extracted using RNeasy Plus Mini Kit (QIAGEN) and converted to cDNA using SuperScript IV VILO Master Mix kit (Invitrogen, Thermo Fisher Scientific), and 25 ng of the template cDNA was then combined with a ready-to-use TaqMan Fast Advanced qPCR Master Mix (Thermo Fisher Scientific) for experiments in a pathway-specific custom TaqMan array (Custom TaqMan Array Fast Plate 96, Life Technologies, Thermo Fisher Scientific). Expression levels of 28 genes relevant to 3 specific pathways - mitochondrial ETC complexes ( $M t$ - $C y b$, $M t-N d 1, M t-N d 2, M t-N d 4, M t-N d 5, M t-A t p 6, M t-A t p 8, M t-C o 1, M t-C o 3, N o x 4, N o s 2)$, oxidative stress and antioxidant defense (Cat, Gpx1, Gpx2, Gsr, Prdx1, Sod1, Sod2, Srxn1, Nqo1, Mt3, Cygb), and hypoxia, stress, and toxicity (Bax, Bcl2l1, Casp8, Hifla, Nfkb1) - were assayed in triplicate along with 4 endogenous housekeeping control genes (18S rRNA, Gapdh, Pgk1, and Actb). To better understand the behavioral differences in P28 offspring, we examined the expression of Nrxn1 in the amygdala, anterior cingulate cortex, and somatosensory cortex of male and female P28 offspring in a separate set of experiments. Thermal cycling was performed in 7500 Fast Real-Time PCR System (Applied Biosystems, Thermo Fisher Scientific), and the threshold cycle values for all genes were calculated using proprietary software. We tested the experimental stability of all 4 endogenous reference genes using the geNorm algorithm and determined Pgk1 to be the most stable reference gene for oxidative stress studies and both Pgk1 and Actb for the learning-associated studies. Relative mRNA expression, normalized either to $P g k 1$ or a geometric mean of $P g k 1$ and $A c t b$, was calculated using the $2^{-\triangle \Delta \mathrm{CT}}$ method with sex-matched control samples as a reference.

Western blotting. Lysates for Western blot were prepared from approximately $100 \mathrm{mg}$ of fetal cortex using RIPA buffer (50 mM Tris- $\mathrm{HCl}$ at $\mathrm{pH}$ 7.5, $150 \mathrm{mM} \mathrm{NaCl}, 2 \mathrm{mM}$ EDTA, 1\% NP-40, 0.1\% SDS) with protease and phosphatase inhibitor cocktail (Thermo Fisher Scientific). Mitochondrial and nuclear fractions from the fetal cortex were separately prepared using appropriate kits (Mitochondrial Isolation Kit, catalog 89801, and Subcellular Protein Fractionation Kit, catalog 87790; Thermo Fisher Scientific) following the manufacturer's instructions. Approximately $30 \mu \mathrm{g}$ of protein was subjected to gel electrophoresis and transferred to membranes using Bolt Western blot reagents from Thermo Fisher Scientific (Bolt 4-12\% Bis-Tris gel, catalog NW04125; Bolt sample reducing agent, catalog B0009; Bolt LDS sample buffer, catalog B0007; iBlot2 dry blotting system). Membranes were blocked with TBST buffer (catalog S1012, EZ Bioresearch) containing 5\% milk for 1 hour at room temperature on a shaker. Following a brief wash with TBST buffer, the membranes were immunoblotted overnight at $4^{\circ} \mathrm{C}$ on a shaker with antibodies against HIF1A (catalog 14179, Cell Signaling Technology) and CASP3 (Asp-175) (catalog 9661, Cell Signaling Technology) and with OXPHOS rodent antibody cocktail (ab110413) (catalog MS604300, Abcam Inc.). For normalization, $\beta$-actin (catalog MA5-11869, Thermo Fisher Scientific), VDAC1 (catalog ab15895, Abcam Inc.), and histone H3 (catalog 9715S, Cell Signaling Technology) were used. For OXPHOS experiments, rat heart mitochondria were used as positive controls. All the primary antibodies were used at a dilution of 1:1000. HRP-conjugated secondary antibodies (anti-rabbit IgG, catalog 7074, and anti-mouse IgG, catalog 7076; Cell Signaling Technology) were used at a dilution of 1:1000 for 1 hour at room temperature on a shaker. Immunoblots were incubated with Western ECL substrate (catalog 1705060, Bio-Rad Inc.) for 5 minutes at room temperature, followed by exposure to film inside a cassette in a dark room, and developed using Konica Minolta, Inc., film processor (catalog SRX-101A). Western blot images were processed with ImageJ (NIH) for densitometric quantification. 
Functional connectivity analysis. Functional connectivity of the brain of OXT- and saline-exposed male offspring was assessed at P23 using a reflectance mode fcOIS imaging system (64). The fcOIS system images the brain through the intact skull and records spontaneous fluctuations in oxy- and deoxyhemoglobin. Through neurovascular coupling, these fluctuations represent spatiotemporal variations in neural activity, similar to human BOLD-fMRI. Briefly, juvenile rats were anesthetized with an i.p. injection of ketamine $(86.9 \mathrm{mg} / \mathrm{kg})$ and xylazine $(13.4 \mathrm{mg} / \mathrm{kg})$ as described previously (65). Optical intrinsic signals were acquired during sequential elimination provided by 4 polarized light-emitting diodes (LEDs, Thorlabs, 470 nm: M470L3-C1, 590 nm: M590L3-C1, 617 nm: M617L3-C1, and $625 \mathrm{~nm}$ : M625L3-C1) placed approximately $20 \mathrm{~cm}$ above the rat's head. Diffuse reflected light from the rat head was detected by a cooled, frame-transfer EMCCD camera (iXon 897 Ultra, Andor Technologies). A crossed linear polarizer was placed in front of the camera lens to reject specular reflection from the LEDs. The LEDs and camera were time-synchronized and controlled via a National Instruments data acquisition card (PCI-6733) and computer (Dell) using custom-written software (MATLAB, MathWorks) at a frame rate of $120 \mathrm{~Hz}$ (30 $\mathrm{Hz} / \mathrm{LED}$ ). Image processing and functional connectivity measurements (including seed-based functional connectivity, homotopic functional connectivity, and regional node degree) were performed as described previously (59). Group averaged correlation maps and matrices are presented in Supplemental Figure 7.

Statistics. For assay and biochemical data, outliers were detected and eliminated using robust regression and outlier analysis with Q set to $10 \%$. Normality of residuals was checked with D'Agostino-Pearson omnibus test. Data with non-normal residuals were Box-Cox transformed before statistical testing. For experiments where sex was not included as a variable (i.e., placental imaging), data were analyzed with either Student's $t$ test or Mann-Whitney $U$ test. For experiments where sex of the offspring was included as a variable, data were analyzed with a 2-way ANOVA followed by Holm-Šidák multiple-comparisons test. RNASeq data were analyzed with generalized linear models to test for gene/transcript-level differential expression. Differentially expressed genes and transcripts were then filtered for FDR-adjusted $P$ values less than or equal to 0.05 . Behavioral data were analyzed with repeated-measures ANOVA models to evaluate effects of treatment, sex, and time-related variables. Following a statistically significant interaction between the main factors, simple main effects were calculated to provide clarification of statistically significant between-treatment and within-treatment differences. Where appropriate, the Huynh-Feldt adjustment was used to protect against violations of sphericity, and Bonferroni's correction was applied to multiple pairwise comparisons. Before statistical testing of fcOIS measures, Pearson's $r$ values were transformed to Fisher's Z-scores followed by a 2-tailed Student's $t$ test. Seed-based comparisons across groups were performed using a 2-tailed Student's $t$ test assuming unequal group variance, followed by FDR correction for multiple comparisons. Maps of node degree were compared at each pixel using a 2-tailed Student's $t$ test assuming unequal group variance and corrected for multiple comparisons using clustering statistics. Differences between groups were considered statistically significant if $P<0.05$ following correction for multiple comparisons. Data are presented as mean \pm SEM. Statistical analyses of biochemical data were performed with Prism for macOS (version 8.2.1, GraphPad Software Inc.), behavioral data with SYSTAT12, and fcOISI data with MATLAB.

Study approval. All experiments reported here were approved by the Institutional Animal Care and Use Committee at Washington University in St. Louis (protocol 20170010) and comply with the Animal Research: Reporting of In Vivo Experiments guidelines.

\section{Author contributions}

AP conceived the study design, planned the experiments, and wrote the manuscript with help from all coauthors. AP, TG, and JJ developed the OXT dosing paradigm and conducted the molecular biology experiments. JDQ and JRG performed the placental imaging experiments, analyzed the data, and helped draft the manuscript. SBC performed the behavioral experiments, and SEM and DFW conducted the analysis and helped with interpretation and with drafting of the manuscript. AB and AQB collected, analyzed, and interpreted the fcOISI data and helped write the manuscript. NR provided the clinical context and assisted with drafting of the final manuscript.

\section{Acknowledgments}

This work was supported by start-up funds from the Department of Anesthesiology, Washington School of Medicine (to AP), and NIH R01 grant NS102870 (to AQB). Placental imaging studies presented in this work were carried out, in part, using the Small Animal MR Facility of the Mallinckrodt Institute of Radiology, 
Washington University in St. Louis (NIH grant R01 HD086323 to JRG and U54 HD087011 to the Intellectual and Developmental Disabilities Research Center at Washington University in St. Louis). Animal behavioral studies were conducted by the Animal Behavior Core, a subcore of the Washington University Intellectual and Developmental Disabilities Research Center (NIH/Eunice Kennedy Shriver National Institute of Child Health and Human Development U54 HD087011). Genomic analysis was conducted at the Genome Technology Access Center (GTAC) in the Department of Genetics at Washington University School of Medicine. GTAC is partially supported by National Cancer Institute Cancer Center Support grant P30 CA91842 to the Siteman Cancer Center; by Institute of Clinical and Translational Sciences/Clinical and Translational Sciences Award grant UL1TR002345 from the National Center for Research Resources, a component of the NIH; and by NIH Roadmap for Medical Research.

Address correspondence to: Arvind Palanisamy, Department of Anesthesiology, Washington University School of Medicine, Campus Box 8054, 660 S. Euclid Ave., St. Louis, Missouri 63110, USA. Phone: 314.362.2628; Email: arvind.palanisamy@wustl.edu. JJ's present address is: Department of Anesthesiology, Beijing Chaoyang Hospital, Capital Medical University, Beijing, China.

1. Kolevzon A, Gross R, Reichenberg A. Prenatal and perinatal risk factors for autism: a review and integration of findings. Arch Pediatr Adolesc Med. 2007;161(4):326-333.

2. Glasson EJ, Bower C, Petterson B, de Klerk N, Chaney G, Hallmayer JF. Perinatal factors and the development of autism: a population study. Arch Gen Psychiatry. 2004;61(6):618-627.

3. Dalman C, Thomas HV, David AS, Gentz J, Lewis G, Allebeck P. Signs of asphyxia at birth and risk of schizophrenia. Population-based case-control study. Br J Psychiatry. 2001;179:403-408.

4. Cannon M, Jones PB, Murray RM. Obstetric complications and schizophrenia: historical and meta-analytic review. Am J Psychiatry. 2002;159(7):1080-1092.

5. Modabbernia A, Mollon J, Boffetta P, Reichenberg A. Impaired gas exchange at birth and risk of intellectual disability and autism: a meta-analysis. J Autism Dev Disord. 2016;46(5):1847-1859.

6. Barkhuizen M, van den Hove DL, Vles JS, Steinbusch HW, Kramer BW, Gavilanes AW. 25 years of research on global asphyxia in the immature rat brain. Neuroscience and biobehavioral reviews. 2017;75: 166-182.

7. Douglas-Escobar M, Weiss MD. Hypoxic-ischemic encephalopathy: a review for the clinician. JAMA Pediatr. 2015;169(4):397-403.

8. Graham EM, Ruis KA, Hartman AL, Northington FJ, Fox HE. A systematic review of the role of intrapartum hypoxia-ischemia in the causation of neonatal encephalopathy. Am J Obstet Gynecol. 2008;199(6):587-595.

9. Koehler RC, Yang ZJ, Lee JK, Martin LJ. Perinatal hypoxic-ischemic brain injury in large animal models: relevance to human neonatal encephalopathy. J Cereb Blood Flow Metab. 2018;38(12):2092-2111.

10. Mallard C, Vexler ZS. Modeling ischemia in the immature brain: how translational are animal models? Stroke. 2015;46(10):3006-3011.

11. van Handel M, Swaab H, de Vries LS, Jongmans MJ. Long-term cognitive and behavioral consequences of neonatal encephalopathy following perinatal asphyxia: a review. Eur J Pediatr. 2007;166(7):645-654.

12. Turner JM, Mitchell MD, Kumar SS. The physiology of intrapartum fetal compromise at term. Am J Obstet Gynecol. 2020;222(1):17-26.

13. Heuser CC, et al. Tachysystole in term labor: incidence, risk factors, outcomes, and effect on fetal heart tracings. Am J Obstet Gynecol. 2013;209(1):32.e1-32.e6.

14. Kunz MK, Loftus RJ, Nichols AA. Incidence of uterine tachysystole in women induced with oxytocin. J Obstet Gynecol Neonatal Nurs. 2013;42(1):12-18.

15. Perrone S, Tataranno LM, Stazzoni G, Ramenghi L, Buonocore G. Brain susceptibility to oxidative stress in the perinatal period. J Matern Fetal Neonatal Med. 2015;28(suppl 1):2291-2295.

16. LaMarca B, Amaral LM, Harmon AC, Cornelius DC, Faulkner JL, Cunningham MW. Placental ischemia and resultant phenotype in animal models of preeclampsia. Curr Hypertens Rep. 2016;18(5):38.

17. Derrick M, Drobyshevsky A, Ji X, Tan S. A model of cerebral palsy from fetal hypoxia-ischemia. Stroke. 2007;38(2 suppl):731-735

18. Ramaekers $\mathrm{CH}$, et al. Hypoxia disrupts the Fanconi anemia pathway and sensitizes cells to chemotherapy through regulation of UBE2T. Radiother Oncol. 2011;101(1):190-197.

19. Scanlon SE, Glazer PM. Hypoxic stress facilitates acute activation and chronic downregulation of fanconi anemia proteins. Mol Cancer Res. 2014;12(7):1016-1028.

20. Apps MA, Rushworth MF, Chang SW. The anterior cingulate gyrus and social cognition: tracking the motivation of others. Neuron. 2016;90(4):692-707.

21. Guo B, et al. Anterior cingulate cortex dysfunction underlies social deficits in Shank3 mutant mice. Nat Neurosci. 2019;22(8):1223-1234

22. Hughes BL, Beer JS. Orbitofrontal cortex and anterior cingulate cortex are modulated by motivated social cognition. Cereb Cortex. 2012;22(6):1372-1381.

23. Mao CV, et al. Pregenual anterior cingulate gyrus involvement in spontaneous social interactions in primates-evidence from behavioral, pharmacological, neuropsychiatric, and neurophysiological findings. Front Neurosci. 2017;11:34.

24. Carrillo M, Han Y, Migliorati F, Liu M, Gazzola V, Keysers C. Emotional mirror neurons in the rat's anterior cingulate cortex. Curr Biol. 2019;29(8):1301-1312.e6. 
25. Hadland KA, Rushworth MF, Gaffan D, Passingham RE. The effect of cingulate lesions on social behaviour and emotion. Neuropsychologia. 2003;41(8):919-931.

26. White BR, Bauer AQ, Snyder AZ, Schlaggar BL, Lee JM, Culver JP. Imaging of functional connectivity in the mouse brain. PLoS One. 2011;6(1):e16322.

27. Ahmed AI, Zhu L, Aldhaheri S, Sakr S, Minkoff H, Haberman S. Uterine tachysystole in spontaneous labor at term. JMatern Fetal Neonatal Med. 2016;29(20):3335-3339.

28. Crane JM, Young DC, Butt KD, Bennett KA, Hutchens D. Excessive uterine activity accompanying induced labor. Obstet Gynecol. 2001;97(6):926-931.

29. Palanisamy A, Lopez J, Frolova A, Macones G, Cahill AG. Association between uterine tachysystole during the last hour of labor and cord blood lactate in parturients at term gestation. Am J Perinatol. 2019;36(11):1171-1178.

30. González-Valenzuela MJ, López-Montiel D, González-Mesa ES. Exposure to synthetic oxytocin during delivery and its effect on psychomotor development. Dev Psychobiol. 2015;57(8):908-920.

31. Hertz-Picciotto I, Schmidt RJ, Krakowiak P. Understanding environmental contributions to autism: causal concepts and the state of science. Autism Res. 2018;11(4):554-586.

32. Kurth L, Haussmann R. Perinatal Pitocin as an early ADHD biomarker: neurodevelopmental risk? J Atten Disord. 2011;15(5):423-431.

33. Modabbernia A, Velthorst E, Reichenberg A. Environmental risk factors for autism: an evidence-based review of systematic reviews and meta-analyses. Mol Autism. 2017;8:13.

34. Smallwood M, Sareen A, Baker E, Hannusch R, Kwessi E, Williams T. Increased risk of autism development in children whose mothers experienced birth complications or received labor and delivery drugs. ASN Neuro. 2016;8(4):1759091416659742.

35. Palanisamy A, et al. Oxytocin alters cell fate selection of rat neural progenitor cells in vitro. PLoS One. 2018;13(1):e0191160

36. Kenkel WM, et al. Behavioral and epigenetic consequences of oxytocin treatment at birth. Sci Adv. 2019;5(5):eaav2244.

37. Boksa P, Zhang Y, Nouel D. Maternal oxytocin administration before birth influences the effects of birth anoxia on the neonatal rat brain. Neurochem Res. 2015;40(8):1631-1643.

38. Demarest TG, Schuh RA, Waddell J, McKenna MC, Fiskum G. Sex-dependent mitochondrial respiratory impairment and oxidative stress in a rat model of neonatal hypoxic-ischemic encephalopathy. J Neurochem. 2016;137(5):714-729.

39. Demarest TG, Schuh RA, Waite EL, Waddell J, McKenna MC, Fiskum G. Sex dependent alterations in mitochondrial electron transport chain proteins following neonatal rat cerebral hypoxic-ischemia. J Bioenerg Biomembr. 2016;48(6):591-598.

40. Thornton C, Jones A, Nair S, Aabdien A, Mallard C, Hagberg H. Mitochondrial dynamics, mitophagy and biogenesis in neonatal hypoxic-ischaemic brain injury. FEBS Lett. 2018;592(5):812-830.

41. Weis SN, et al. Neonatal hypoxia-ischemia induces sex-related changes in rat brain mitochondria. Mitochondrion. 2012;12(2):271-279.

42. Dachtler J, Ivorra JL, Rowland TE, Lever C, Rodgers RJ, Clapcote SJ. Heterozygous deletion of $\alpha$-neurexin I or $\alpha$-neurexin II results in behaviors relevant to autism and schizophrenia. Behav Neurosci. 2015;129(6):765-776.

43. Grayton HM, Missler M, Collier DA, Fernandes C. Altered social behaviours in neurexin $1 \alpha$ knockout mice resemble core symptoms in neurodevelopmental disorders. PLoS One. 2013;8(6):e67114.

44. Hu Z, Xiao X, Zhang Z, Li M. Genetic insights and neurobiological implications from NRXN1 in neuropsychiatric disorders Mol Psychiatry. 2019;24(10):1400-1414.

45. Kim HG, et al. Disruption of neurexin 1 associated with autism spectrum disorder. Am J Hum Genet. 2008;82(1):199-207.

46. Adolphs R. What does the amygdala contribute to social cognition? Ann N Y Acad Sci. 2010;1191:42-61.

47. Han Y, et al. Bidirectional cingulate-dependent danger information transfer across rats. PLoS Biol. 2019;17(12):e3000524.

48. Jones CE, et al. Early-life sleep disruption increases parvalbumin in primary somatosensory cortex and impairs social bonding in prairie voles. Sci Adv. 2019;5(1):eaav5188.

49. Twining RC, Vantrease JE, Love S, Padival M, Rosenkranz JA. An intra-amygdala circuit specifically regulates social fear learning. Nat Neurosci. 2017;20(3):459-469.

50. Diaz-Castro J, et al. Gender specific differences in oxidative stress and inflammatory signaling in healthy term neonates and their mothers. Pediatr Res. 2016;80(4):595-601.

51. Giordano G, Tait L, Furlong CE, Cole TB, Kavanagh TJ, Costa LG. Gender differences in brain susceptibility to oxidative stress are mediated by levels of paraoxonase-2 expression. Free Radic Biol Med. 2013;58:98-108.

52. Lavoie JC, Tremblay A. Sex-specificity of oxidative stress in newborns leading to a personalized antioxidant nutritive strategy. Antioxidants (Basel). 2018;7(4):E49.

53. Netto CA, Sanches E, Odorcyk FK, Duran-Carabali LE, Weis SN. Sex-dependent consequences of neonatal brain hypoxiaischemia in the rat. J Neurosci Res. 2017;95(1-2):409-421.

54. Taskiran D, Nesil T, Alkan K. Mitochondrial oxidative stress in female and male rat brain after ex vivo carbon monoxide treatment. Hum Exp Toxicol. 2007;26(8):645-651.

55. Fernandez A, et al. Mitochondrial dysfunction leads to cortical under-connectivity and cognitive impairment. Neuron 2019;102(6):1127-1142.e3

56. Fox MD, Snyder AZ, Vincent JL, Corbetta M, Van Essen DC, Raichle ME. The human brain is intrinsically organized into dynamic, anticorrelated functional networks. Proc Natl Acad Sci U S A. 2005;102(27):9673-9678.

57. Greicius MD, Krasnow B, Reiss AL, Menon V. Functional connectivity in the resting brain: a network analysis of the default mode hypothesis. Proc Natl Acad Sci U S A. 2003;100(1):253-258.

58. Dyson A, et al. Antihyperalgesic properties of the cannabinoid CT-3 in chronic neuropathic and inflammatory pain states in the rat. Pain. 2005;116(1-2):129-137.

59. Hakon J, et al. Multisensory stimulation improves functional recovery and resting-state functional connectivity in the mouse brain after stroke. Neuroimage Clin. 2018;17:717-730.

60. Carey LM, Abbott DF, Lamp G, Puce A, Seitz RJ, Donnan GA. Same intervention-different reorganization: the impact of lesion location on training-facilitated somatosensory recovery after stroke. Neurorehabil Neural Repair. 2016;30(10):988-1000.

61. Scremin AM, Scremin OU, Brechner T. Survival under hypoxia. Age dependence and effect of cholinergic drugs. Stroke. 
1980;11(5):548-552

62. Ortuño JE, Ledesma-Carbayo MJ, Simões RV, Candiota AP, Arús C, Santos A. DCE@urLAB: a dynamic contrast-enhanced MRI pharmacokinetic analysis tool for preclinical data. BMC Bioinformatics. 2013;14:316

63. Maloney SE, et al. Repeated neonatal isoflurane exposures in the mouse induce apoptotic degenerative changes in the brain and relatively mild long-term behavioral deficits. Sci Rep. 2019;9(1):2779.

64. Bauer AQ, Kraft AW, Wright PW, Snyder AZ, Lee JM, Culver JP. Optical imaging of disrupted functional connectivity following ischemic stroke in mice. Neuroimage. 2014;99:388-401.

65. Mitra A, et al. Spontaneous infra-slow brain activity has unique spatiotemporal dynamics and laminar structure. Neuron. 2018;98(2):297-305.e6. 